\title{
EHPBS: Energy Harvesting Prediction Based Scheduling in Wireless Sensor Networks
}

\author{
Berk Akgün \\ Electrical and Electronics Engineering Department \\ Middle East Technical University \\ Ankara, TURKEY
}

\author{
Irmak Aykın \\ Electrical and Electronics Engineering Department \\ Bilkent University \\ Ankara, TURKEY
}

\begin{abstract}
The clustering algorithms designed for traditional sensor networks have been adapted for energy harvesting sensor networks (EHWSN). However, in these algorithms, the intracluster MAC protocols to be used were either not defined at all or they were TDMA based. These TDMA based MAC protocols are not specified except for the fact that cluster heads assign time slots to their members in a random manner. In this paper, we will modify this TDMA based scheduling as follows: members will request a time slot depending on their energy prediction and the cluster heads will assign these slots to members. This method will increase the network lifetime. The proof will be given with simulations.
\end{abstract}

Keywords-Energy Harvesting; Sensor Networks; Clustering; Environmental Energy; TDMA.

\section{INTRODUCTION}

$\mathrm{T}$ oday, wireless sensor networks are widely used and their area of usage increase day by day. They are commonly used in both military and civil applications such as target detection, weather and environmental monitoring and natural disaster prevention. Wireless sensor nodes are tiny and their physical qualifications are very limited. Thus, their batteries should be used efficiently. They consist of 3 parts, namely, processing unit, sensing unit and transmission unit. Compared to others, transmission unit has the major impact on battery lifetime. In the traditional wireless sensor networks, minimizing the energy usage was the main purpose. However, even with the best algorithms, the batteries had a limited lifetime and after that the sensor nodes were dead. Collecting dead nodes and replacing their batteries was not practical. That became an inspiration for energy harvesting sensor networks. With their emerging, the main purpose of sensor networks became efficiently using the available and approximated energy, and preventing sensor nodes from dying.

Then, what are the things to consider when we are designing an energy harvesting sensor network rather than a traditional sensor network? When recharge opportunities are in presence, routing metrics should consider current and future energy levels on top of conventional metrics such as hop count and delivery probability. For instance, a routing protocol can choose a path with nodes expecting to harvest energy soon, rather than a path which consists of nodes with higher existent energy levels. Hence, recharge opportunities can be used for better performances [1]. The main objective is to maximize the collected data given the rate of energy that can be harvested from the medium instead of maximizing the lifetime of the sensor network.

Clustering algorithms are the most widely used network layer protocols due to their load balancing and scalability properties. In clustering algorithms, cluster heads assigns time slots (TDMA) for each member of the cluster. These time slots are not assigned according to a pattern that considers available energy. What we want to do in this paper is to make these assignments according to the energy predictions. In other words, cluster heads will assign these time slots according to the requests from cluster members, and the requests will be made according to the energy predictions. Thus, when a cluster member wakes up and starts harvesting energy, it will also be able to transmit, since its time slot will be assigned accordingly. This way, nodes will not waste their energy trying to recharge their battery. Instead, they will use the harvested energy instantly, as much as possible. Thus, not only longer lifetime duration of wireless sensor networks but also maximum task performance will be achieved. Furthermore, to our knowledge, there is no previous work addressing TDMAbased MAC protocols for EHWSN.

In this paper, we will provide simulation results. The paper briefly discusses the related work in the second chapter, while in the third chapter, the proposed scheme is introduced. In chapter IV, the simulation setup will be explained and finally in chapter $\mathrm{V}$ the results will be discussed.

\section{RELATED WORK}

[2] assumes that periodic energy sources are available and the energy profiles can be estimated perfectly. [3] considers an extended class of energy sources. Also, its algorithm reduces the variance of the duty cycle. Both papers design duty cycles that adapt dynamically to a node in order to achieve maximum efficiency considering energy neutrality operation. [4] and [5] are clustering algorithms designed for wireless sensor networks, namely, LEACH and HEED. [6] suggests an environmental energy harvesting framework in order to use the energy predictions in LEACH and HEED. [7] further introduces an enhanced framework for these. However, the intra-clustering MAC algorithms are not defined by means of energy prediction in these papers. Duty cycling based MAC protocols in wireless sensor networks can be investigated in 2 groups, namely, CSMA-based MAC protocols and TDMAbased MAC protocols. Both of these schemes are energy efficient for wireless sensor networks, but there is no work 
regarding the energy harvester feature. The main difference between these papers is that some of them [8], [9], [10] need time-synchronization and some of them [11], [12], [13] do not.

\section{PROPOSED SCHEME}

\section{A. Battery}

When we consider leakage power, if we assume constant type, age and temperature, batteries leak most right after being charged, according to [14]. Also, we see from [2] that we achieve

$$
\begin{aligned}
& B_{0}+\eta \int_{0}^{T}\left[P_{s}(t)-P_{c}(t)\right]^{+} d t-\int_{0}^{T}\left[P_{c}(t)-P_{s}(t)\right]^{+} d t- \\
& \int_{0}^{T} P_{\text {leak }} d t \geq 0
\end{aligned}
$$

using the rule of energy conservation. Here, $P_{s}(t)$ is defined as the energy harvested from the medium at time $t . P_{c}(t)$ is, on the other hand, the energy being consumed by the load at that time. Round-trip efficiency, the ratio of the energy stored into the battery to the energy brought to the battery, is denoted as $\eta$ and constant leakage power is denoted as $P_{\text {leak }} . B_{0}$ is the current power level of the battery. From this formula, we understand that some of the energy is wasted during the process of battery charging, since the round trip efficiency of the buffer $\eta$ is strictly less than 1 . Thus, if we spend the harvested energy without charging the battery, we exploit the situation and decrease wasted power. To find the value of $\eta$ to be used in calculations, we should investigate the battery type in more detail. NiMH and Li-based batteries are good selections to be used in energy harvesting nodes. Table I summerizes that Li-based batteries have a lot of advantages over others such that they provide high output voltage and energy density while they waste almost no energy due to their high round trip efficiency. Nevertheless, lithium batteries need pulsecharging to be recharged and an extra battery or a charging circuit is needed for that. Conversely, NiMH batteries can be trickle charged. In other words, they can be connected to an energy source directly, and do not require detailed pulse charging circuits. Their energy and power density values are also fairly well. Supercapacitors are also an alternative; however, due to their high leakage, they are not used alone. They are combined with NiMH batteries in some cases [1]. So from Table I, we see that the value for $\eta$ would be 0.66 for most sensor networks, since NiMH is the most widely used battery in solar energy applications. We used solar energy as our energy source due to the ease of predictability of the energy profiles. Also, there is more information regarding solar energy profiles in the literature.

\section{B. Energy Prediction}

When estimating the energy in day $n$ slot $i$, we use the following formula:

$$
E_{e s t 1}(n, i)=\sum_{k=0}^{n-1} \alpha(1-\alpha)^{k} E_{g e n}(n-(k+1), i)
$$

where we take $\alpha$ as 0.2 , which is found to be the optimum value as seen from Fig. 1. Here, $E_{\text {est }}$ is the energy forecasted in a succeeding slot; $E_{\text {gen }}$ is the value of the generated energy in that interval and $\alpha$ is the filter coefficient.

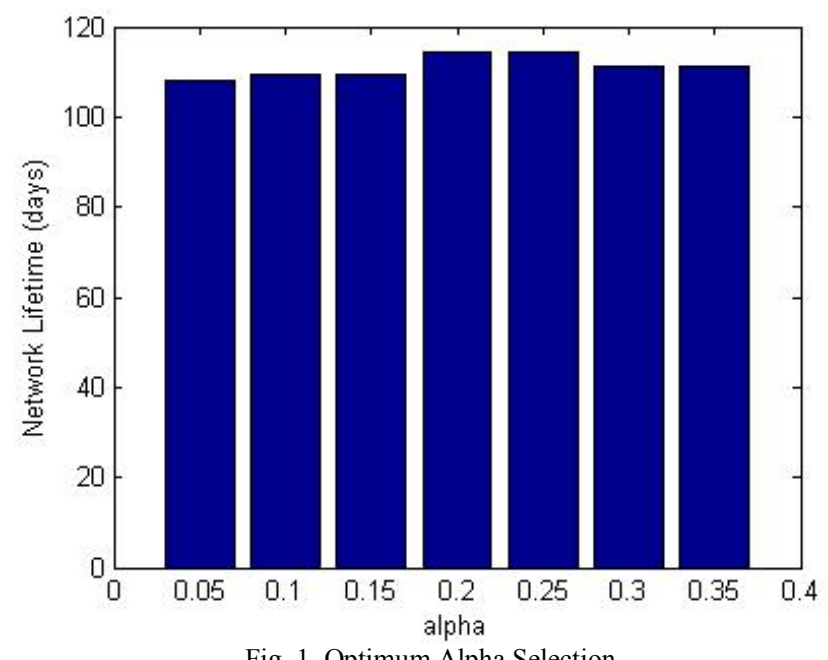

Fig. 1. Optimum Alpha Selection

When calculating the $E_{\text {estl }}(n, i)$, we consider last 10 days' energy generation values due to memory limitations. However, a given day may have an unexpectedly different weather than other days. Thus, to improve the estimated energy more, we calculate the ratio of the summation of energy generation values to summation of energy estimation values in that given day up to slot $\mathrm{i}$. Then the equation becomes as follows:

$$
E_{e s t}(n, i)=E_{e s t 1}(n, i) \times\left(\frac{\sum_{k=0}^{i-1} E_{g e n}(n, k)}{\sum_{k=0}^{i-1} E_{e s t 1}(n, k)}\right)
$$

Using $E_{\text {est }}(n, i)$ and $E_{\text {res }}$, residual energy, values we achieve the parameter

$$
E(n, i)=w_{1}\left(E_{\text {est }}(n, i)\right)+w_{2} E_{\text {res }}
$$

where $w_{1}=w_{2}=1$.

\section{C. $E H P B S$}

In the regular scheme (the intra-cluster communication protocol of LEACH), the operation consists of frames, where one transmission slot per frame is allocated to member nodes. Since the duration of these slots are the same, the number of frames depends on the number of member nodes. In this system, cluster head assigns slots to members randomly, in a TDMA based manner. That is, cluster head doesn't consider any efficiency parameter when allocating the slots [4].

In our scheme, all nodes have data to transmit as in LEACH, so TDMA is more bandwidth efficient than other approaches. To further improve the existing TDMA protocol, we developed EHPBS. In EHPBS, all the members compute the $E_{\text {est }}(n, i)$ value for each slot and send this and $E_{\text {res }}$ values to the cluster head. When $E(n, i)$ value exceeds the battery capacity, the algorithm selects the node with the largest exceeding energy. If there aren't any such nodes, when $E(n, i)$ value is above the power to be consumed, cluster head assigns the slot to the node with largest $E_{e s t}(n, i)$. However, this is only valid for the morning time as the nodes will harvest energy only in mornings. Otherwise, the slot is given to the node with 
TABLE 1[1]

COMPARISON OF RECHARGEABLE BATTERY TECHNOLOGIES

\begin{tabular}{|l|l|l|l|l|l|l|l|l|l|}
\hline $\begin{array}{l}\text { Battery } \\
\text { Type }\end{array}$ & $\begin{array}{l}\text { Nominal } \\
\text { Voltage }\end{array}$ & Capacity & $\begin{array}{l}\text { Weight } \\
\text { Energy } \\
\text { Density } \\
(\mathbf{W h} / \mathbf{k g})\end{array}$ & $\begin{array}{l}\text { Power } \\
\text { Density } \\
(\mathbf{W} / \mathbf{k g})\end{array}$ & $\mathbf{E f f i c i e n c y}$ & $\begin{array}{l}\text { Self } \\
\text { Discharge }\end{array}$ & $\begin{array}{l}\text { Memory } \\
\text { Effect? }\end{array}$ & $\begin{array}{l}\text { Charging } \\
\text { Method } \\
(\boldsymbol{\%} / \mathbf{m o n t h})\end{array}$ & $\begin{array}{l}\text { Recharge } \\
\text { Cycles }\end{array}$ \\
\hline \hline SLA & 6 & 1300 & 26 & 180 & $70-92$ & 20 & No & Trickle & $500-800$ \\
\hline NiCd & 1.2 & 1100 & 42 & 150 & $70-90$ & 10 & Yes & Trickle & 1500 \\
\hline NiMH & 1.2 & 2500 & 100 & $250-1000$ & 66 & 20 & No & Trickle & 1000 \\
\hline Li-ion & 3.7 & 740 & 165 & 1800 & 99.9 & $<10$ & No & Pulse & 1200 \\
\hline Li-polymer & 3.7 & 930 & 156 & 3000 & 99.8 & $<10$ & No & Pulse & $500-1000$ \\
\hline
\end{tabular}

largest $E_{\text {res }}$. However, when $E(n, i)$ values are not large enough, since the nodes may die while transmitting even when they are harvesting, the channel is given to the node with largest $E(n, i)$ value. When the slot is assigned to a node and if it is using its residual energy, its $E_{\text {res }}$ value is decreased by the amount that is used. This way, we're planning to assign the channel to the node that is harvesting most. Thus, the wasted power will be decreased, since harvested energy will be used to transmit without charging the battery. Since no slot is skipped without transmission, both schemes will have the same throughput. When the channel is assigned to a node, others will be in sleep mode. Here, we do not deal with the fairness since it is not an important issue in sensor networks. The more important part is to monitor the tasks, thus more slots may be allocated to some nodes than others.

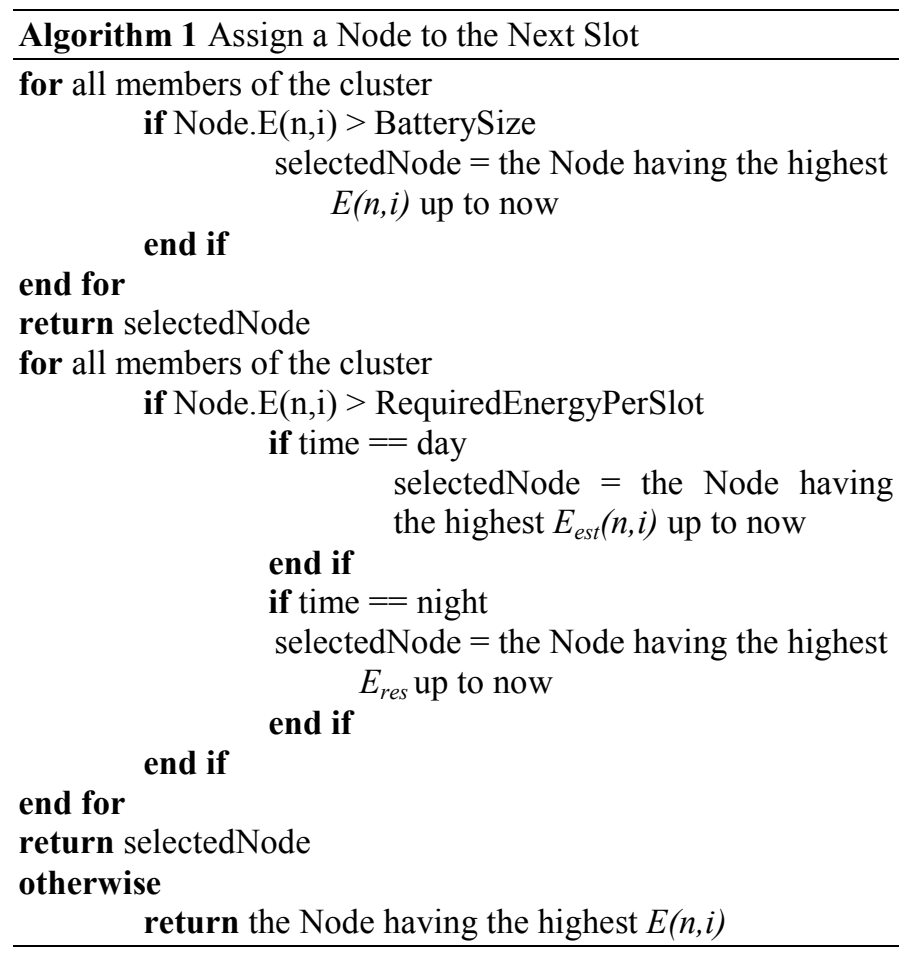

\section{Simulation SetuP}

We assumed that the cluster head is selected according to LEACH. The simulation environment is $\mathrm{C}++$. Doing the simulations with more advanced programs would definitely give more accurate results; however, since we wanted to compare two algorithms instead of getting specific numerical results, $\mathrm{C}++$ worked for us and we were able to get percentile

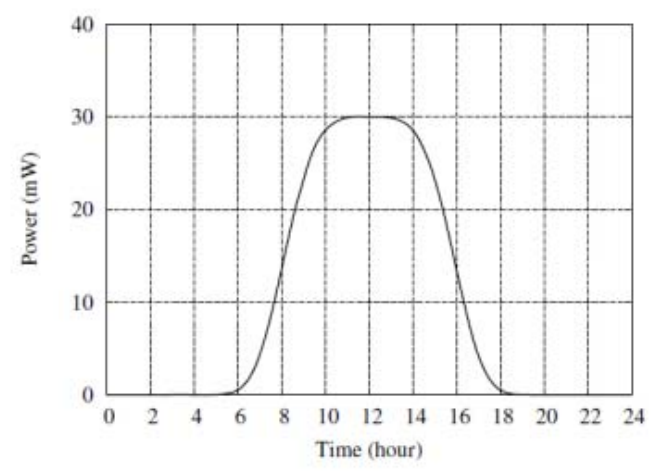

Fig. 2. Generating Power [7]

TABLE 2 [7]

Weather Parameter

\begin{tabular}{|c|c|c|}
\hline type & prob & $R_{\text {weather }}$ \\
\hline \hline FINE & 0.2 & $0.8-1.0$ \\
\hline CLEAR & 0.3 & $0.6-0.8$ \\
\hline CLOUDY & 0.3 & $0.15-0.5$ \\
\hline RAINY & 0.2 & $0.05-0.2$ \\
\hline
\end{tabular}

improvements. We have compared the performances of the regular algorithm and our scheme in terms of network lifetime. As the regular algorithm, we took the intra-cluster communication algorithm of LEACH since many other clustering algorithms also use that method. We expected to decrease the wasted power caused by the round-trip efficiency and thus increase the network lifetime with our proposed scheme. Synchronization problem has not been considered since it's outside the scope of this paper. Nodes were assumed to have packets to send all the time. We assumed that a node dies when its unrechargeable battery becomes empty.

We have set some simulation parameters as follows; the time between two consecutive cluster setup phases $=60$ minutes, slot duration $=30$ seconds, initial energy of the rechargeable battery $=50 \mathrm{~J}$, initial energy of the unrechargeable battery $=100 \mathrm{~J}$, power consumption in active $\operatorname{mode}=38 \mathrm{~mW}$ and power consumption in sleep mode $=30 \mathrm{uW}$. We neglected the effect of leakage since it is the same in both schemes.

To calculate the generating power of solar cell, we use [7]

$$
P_{\text {charge }}(t)=R_{\text {weather }} \times R_{\text {position }} \times f(t)
$$

$f(t)$ is the maximum power generation ability of the solar cell as a function of time. If $R_{\text {weather }}$, determined according to Table II and stays the same throughout the day, and $R_{\text {position }}$, depending on the position of the node, namely sun and shade, get their 


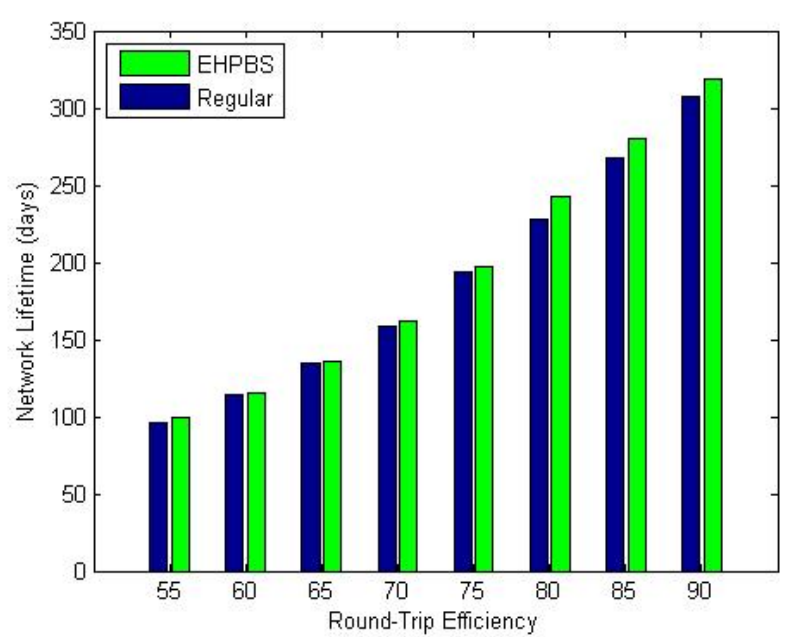

Fig. 3. Network Lifetime Comparison for Different Round-Trip Efficiencies

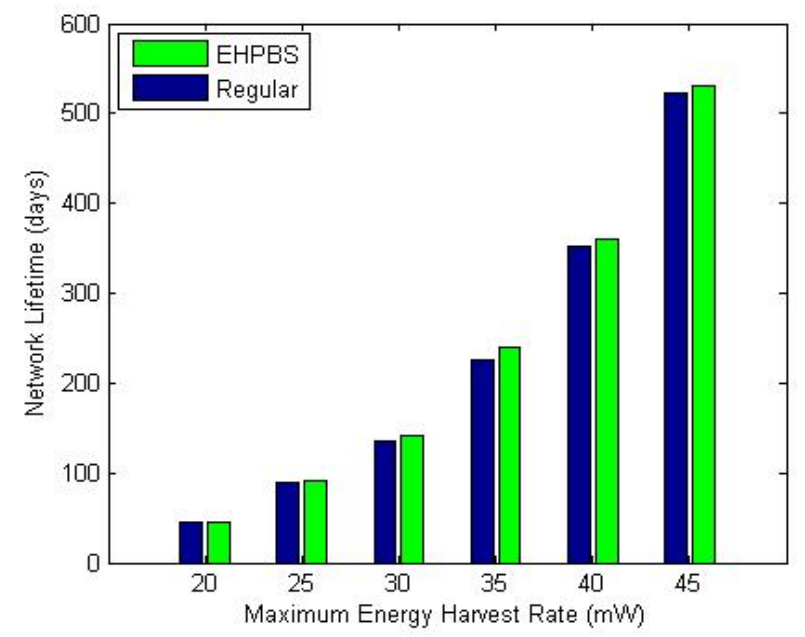

Fig. 4. Network Lifetime Comparison for Different Energy Harvest Rates

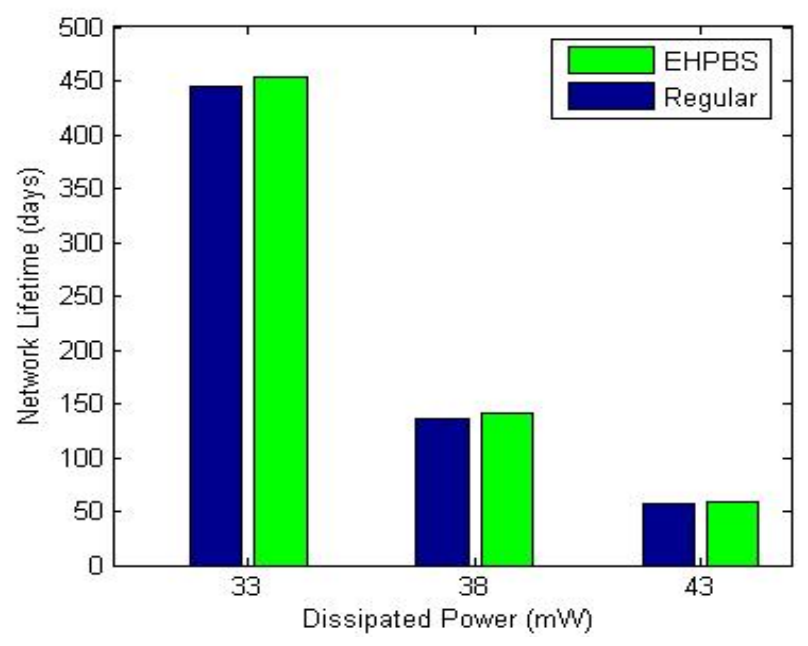

Fig. 5. Network Lifetime Comparison for Different Dissipated Powers

maximum values, the highest power generation is achieved [7]. According to these values, a node can generate at most $30 \mathrm{~mW}$.

\section{RESUlTS}

Network lifetime is defined as the time spent until the first node dies. EHPBS and the regular scheme have been compared in many different topologies. The simulation was run 100 times for each data and their average was taken in order to find the most accurate in accordance with Central Limit Theorem. In addition to the round-trip efficiency of $\mathrm{NiMH}$ battery, we have also investigated the performance of EHPBS for different round trip efficiencies. As seen from Fig.3, EHPBS performs better than the regular scheme for all round-trip efficiencies, but the difference between algorithms increases with increasing round trip efficiencies. However, the percentage of the improvement is nearly the same and it is around $3.76 \%$. This figure with various round-trip efficiencies would be very helpful if the battery type of the sensors change in the future. From Fig.4 we can see that network lifetime increases with increasing energy harvest rate, since when the nodes are able to harvest more energy, batteries last longer and the time spent until the death of first node increases. Here, average improvement compared to regular scheme is $2.38 \%$. We did this comparison since the maximum energy harvest rate can change according to the season or the geographical region of the sensors and the generating value of the solar cell depends on this parameter. Finally, as seen from Fig.5, as the dissipated power increases, network lifetime decreases, since the batteries die sooner. The average gain that EHPBS provides is $2.25 \%$. It is important to see the change in the network lifetime with respect to various dissipated power values since different transceivers have different characteristics.

\section{CONCLUSION}

As explained before, the main purpose of EHWSN is to increase the network lifetime and prevent nodes from dying. When there is a possibility of recharging the battery, routing metrics should regard current and future energy levels as well as classical metrics like delivery probability and hop count. In this paper, we tried to propose a scheme that exploits battery round-trip efficiency. As the energy source, we used solar energy since it is easier to predict compared to other energy sources such as vibration and pressure. We used $\mathrm{NiMH}$ batteries in our calculations since they do not require complicated circuits to be recharged. Moreover, their power and energy density values are quite well. Since batteries leak most right after being charged and also some of the harvested energy is wasted during the process of battery charging, we developed a scheme that will allow the node to transmit packets right after energy harvesting. In the regular scheme, cluster head assigns slots to members randomly, in a TDMA based manner. In our scheme, the channel is assigned to the node that harvests most energy at that time, given that its total energy is above the power to be consumed. If not, another node with higher total energy is allowed to transmit. This way, network lifetime was able to be increased.

As the future work, we are planning to simulate the system in more detailed simulation programs like NS3 and implement it on a real sensor network. We will also compare the algorithm with MAC protocols other than LEACH's. Furthermore, we aim to extend the algorithm by changing the energy source 
from solar to vibration or pressure. That way, we will have covered a much broader category of energy harvesting wireless sensor networks.

\section{REFERENCES}

[1] Sudevalayam and Kulkarni, "Energy Harvesting Sensor Nodes: Survey and Implications" in IEEE Communications Surveys \& Tutorials, vol. 13, no. 3, Third Quarter 2011.

[2] J. Hsu, S. Zahedi, A. Kansal, M. Srivastava, and V. Raghunathan, "Adaptive Duty Cycling for Energy Harvesting Systems," in Proc. 2006 International Symposium on Low Power Electronics and Design. ACM, 2006, pp. 180-185.

[3] C. Vigorito, D. Ganesan, and A. Barto, "Adaptive Control of Duty Cycling in Energy-Harvesting Wireless Sensor Networks," 4th Annual IEEE Communications Society Conference on Sensor, Mesh and Ad Hoc Communications and Networks., pp. 21-30, June 2007.

[4] W. Heinzelman, A. Chandrakasan, and H. Balakrishnan, "An Application-Specific Protocol Architecture for Wireless Microsensor Networks," IEEE Trans. Wireless Commun., vol. 1, no. 4, Oct. 2002, pp. $660-70$.

[5] 0. Younis and S. Fahmy, "Distributed Clustering in Ad Hoc Sensor Networks: A Hybrid, Energy-Efficient Approach," Proc. IEEE INFOCOM, Hong Kong, Mar. 2004; an extended version appeared in IEEE Trans. Mobile Comp., vol. 3, no. 4, Oct.-Dec. 2004, pp. 366-79.

[6] A. Kansal, et. al., "An Environmental Energy Harvesting Framework for Sensor Networks," ACM International Symposium on Low Power Electronics and Design, pp. 481-486, 2003.

[7] K. Kinoshita, T. Okazaki, H. Tode, and K. Murakami, "A Data Gathering Scheme for Environmental Energy-Based Wireless Sensor Networks," in 5th IEEE Consumer Communications and Networking Conference., Jan. 2008, pp. 719-723.

[8] W. Ye, J. Heidemann, and D. Estrin, "An Energy-efficient MAC Protocol for Wireless Sensor Networks," in INFOCOM 2002. Proc. Twenty-First Annual Joint Conference of the IEEE Computer and Communications Societies., vol. 3, 2002, pp. 1567-1576.

[9] A. Rowe, R. Mangharam, and R. Rajkumar, "RT-Link: A Global TimeSynchronized Link Protocol for Sensor Networks," Ad Hoc Networks, vol. 6, no. 8, pp. 1201-1220, 2008.

[10] T. van Dam and K. Langendoen, "An adaptive energy-efficient MAC protocol for wireless sensor networks," in SenSys '03: Proc. 1st International Conference on Embedded Networked Sensor Systems. New York, NY, USA: ACM, 2003, pp. 171-180.

[11] Y. Sun, O. Gurewitz, and D. B. Johnson, "RI-MAC: A Receiver-Initiated Asynchronous Duty Cycle MAC Protocol for Dynamic Traffic Loads in Wireless Sensor Networks," in SenSys '08: Proc. 6th ACM Conference on Embedded Network Sensor Systems. New York, NY, USA: ACM, 2008, pp. 1-14.

[12] J. Polastre, J. Hill, and D. Culler, "Versatile Low Power Media Access for Wireless Sensor Networks," in Proc. 2nd International Conference on Embedded Networked Sensor Systems. ACM, 2004, pp. 95-107.

[13] M. Buettner, G. V. Yee, E. Anderson, and R. Han, "X-MAC: A Short Preamble MAC Protocol for Duty-cycled Wireless Sensor Networks," in Proc. 4th International Conference on Embedded Networked Sensor Systems. ACM, 2006, pp. 307-320.

[14] B. Devillers and D. Gunduz, "Energy harvesting communication system with battery constraint and leakage," in preparation, 2011. 